\title{
ON CONVERGENCE OF SUBSPACES GENERATED BY DILATIONS OF POLYNOMIALS. AN APPLICATION TO BEST LOCAL APPROXIMATION
}

\author{
FABIÁN E. LEVIS AND CLAUDIA V. RIDOLFI
}

\begin{abstract}
We study the convergence of a net of subspaces generated by dilations of polynomials in a finite dimensional subspace. As a consequence, we extend the results given by Zó and Cuenya [Advanced Courses of Mathematical Analysis II (Granada, 2004), 193-213, World Scientific, 2007] on a general approach to the problems of best vector-valued approximation on small regions from a finite dimensional subspace of polynomials.
\end{abstract}

\section{INTRODUCTION}

Suppose that $\left\{a_{j}\right\}$ is a data set. These data are values of a function and its derivatives at a point. If we want to approximate these data using a polynomial of degree at most $l$, which will be the best algorithm to use? A Taylor polynomial of degree $l$ is probably the most natural procedure to use.

The problem of finding an optimal algorithm to approximate a finite number of data corresponding to a function is developed in the best local approximation theory.

In 1934, Walsh proved in [11 that the Taylor polynomial of degree $l$ for an analytic function $f$ can be obtained by taking the limit as $\epsilon \rightarrow 0$ of the best Chebyshev approximation to $f$ from $\Pi^{l}$ on the disk $|z| \leq \epsilon$. This paper was the first association between the best local approximation to a function $f$ from $\Pi^{l}$ in 0 and the Taylor polynomial for $f$ at the origin. However, the concept of best local approximation has been introduced and developed more recently by Chui, Shisha, and Smith in [2. Later, several authors [3, 4, 5, 6, 8, 9, 10, 12, have studied this problem.

We consider a family of function seminorms $\left\{\|\cdot\|_{\epsilon}\right\}_{\epsilon>0}$, acting on Lebesgue measurable functions $F: B \subset \mathbb{R}^{n} \rightarrow \mathbb{R}^{k}$, where $B$ is the unit ball centered at the origin in $\mathbb{R}^{n}$. We will use the notation $F^{\epsilon}(x)=F(\epsilon x)$ and $\|F\|_{\epsilon}^{*}=\left\|F^{\epsilon}\right\|_{\epsilon}$. For $l \in \mathbb{N} \cup\{0\}$,

2010 Mathematics Subject Classification. Primary 40A05, 41A10, 41A65.

Key words and phrases. Convergence of subspaces; Best local approximation; Abstract norms; Homogeneous dilations.

This paper was partially supported by Universidad Nacional de Río Cuarto (grant PPI 18/C472), Universidad Nacional de La Pampa, Facultad de Ingeniería (grant Resol. Nro. 165/18), and CONICET (grant PIP 112-201501-00433CO). 
we will denote by $\Pi^{l}$ the class of algebraic polynomials in $n$ variables of degree at most $l$, and $\Pi_{k}^{l}$ the set $\left\{P=\left(p_{1}, \ldots, p_{k}\right): p_{s} \in \Pi^{l}\right\}$.

Let $\mathcal{A}$ be a subspace of $\Pi_{k}^{l}$ and let $\left\{P_{\epsilon}\right\}_{\epsilon>0}$ be a net of best approximants to $F$ from $\mathcal{A}$ with respect to $\|\cdot\|_{\epsilon}^{*}$, i.e.,

$$
\left\|F-P_{\epsilon}\right\|_{\epsilon}^{*} \leq\|F-P\|_{\epsilon}^{*}, \quad \text { for all } P \in \mathcal{A} \text {. }
$$

If the net $\left\{P_{\epsilon}\right\}_{\epsilon>0}$ has a limit in $\mathcal{A}$ as $\epsilon \rightarrow 0$, this limit is called the best local approximation to $F$ from $\mathcal{A}$ in 0 . According to $\left[1.1\right.$, we observe that $P_{\epsilon}^{\epsilon}$ is a polynomial in

$$
\mathcal{A}^{\epsilon}:=\left\{P^{\epsilon}: P \in \mathcal{A}\right\} \subset \Pi_{k}^{l}
$$

of best approximation to $F^{\epsilon}$ by elements of the class $\mathcal{A}^{\epsilon}$, with respect to the seminorm $\|\cdot\|_{\epsilon}$. We write it briefly as $P_{\epsilon}^{\epsilon} \in \mathcal{P}_{\mathcal{A}^{\epsilon}, \epsilon}\left(F^{\epsilon}\right)$. Note that $\mathcal{A}^{\epsilon}$ is a subspace generated by dilations of polynomials in $\mathcal{A}$.

From now on, we assume the following properties for the family of function seminorms $\|\cdot\|_{\epsilon}, 0 \leq \epsilon \leq 1$.

(1) For $F=\left(f_{1}, \ldots, f_{k}\right)$ and $G=\left(g_{1}, \ldots, g_{k}\right)$, we have $\|F\|_{\epsilon} \leq\|G\|_{\epsilon}$, for every $\epsilon>0$, whenever $\left|f_{s}\right| \leq\left|g_{s}\right|, s=1, \ldots, k$.

(2) If 1 is the function $F(x)=(1, \ldots, 1)$, we have $\|1\|_{\epsilon}<\infty$, for all $\epsilon>0$.

(3) For every $F \in C_{k}(B)$, we have $\|F\|_{\epsilon} \rightarrow\|F\|_{0}$, as $\epsilon \rightarrow 0$, where $C_{k}(B)$ is the set of continuous functions $F: B \subset \mathbb{R}^{n} \rightarrow \mathbb{R}^{k}$. Moreover, $\|\cdot\|_{0}$ is a norm on $C_{k}(B)$.

An important point to note here is that there exist positive constants $C=$ $C(m, k)$ and $\epsilon(m, k)$ such that for every $0<\epsilon \leq \epsilon(m, k)$,

$$
\frac{1}{C}\|P\|_{0} \leq\|P\|_{\epsilon} \leq C\|P\|_{0}, \quad \text { for every } P \in \Pi_{k}^{m}
$$

[13, Proposition 3.1].

In order to give an example of norms $\|\cdot\|_{\epsilon}, 0 \leq \epsilon \leq 1$, with the properties (1)-(3), we recall a definition of convergence of measures given in [6]. See also [1] for the notion of weak convergence of measures in general.

Definition 1.1. Let $\mu_{\epsilon}, 0 \leq \epsilon \leq 1$, be a family of probability measures on B. We say that the measures $\mu_{\epsilon}$ converge weakly in the proper sense to the measure $\mu_{0}$ if we have

$$
\int_{B} f(x) d \mu_{\epsilon}(x) \rightarrow \int_{B} f(x) d \mu_{0}(x), \quad f \in C_{1}(B),
$$

and $\mu_{0}\left(B^{\prime}\right)>0$ for any ball $B^{\prime} \subset B$.

The assumption on the measure $\mu_{0}$ implies that

$$
\|F\|_{\epsilon}=\|F\|_{L^{p}\left(\mu_{\epsilon}\right)}=\left(\int_{B}\|F\|^{p} d \mu_{\epsilon}\right)^{\frac{1}{p}}
$$

is actually a norm on $C_{k}(B)$ for $\epsilon=0$ and $1 \leq p<\infty$, where $\|\cdot\|$ stands for any monotone norm on $R^{k}$. We use a monotone norm on $R^{k}$ to ensure property (1) for the family of seminorms $\|\cdot\|_{\epsilon}, 0 \leq \epsilon \leq 1$. 
Let $F$ be in $C_{k}(B)$; it is readily seen, by using the definition of weak convergence of measures, that there exists $\epsilon_{0}=\epsilon_{0}(F)>0$ such that if $\|F\|_{\epsilon}=\|F\|_{L^{p}\left(\mu_{\epsilon}\right)}=0$, for some $0<\epsilon \leq \epsilon_{0}$, then $F=0$. Moreover we have that $\|F\|_{\epsilon}=\|F\|_{L^{p}\left(\mu_{\epsilon}\right)}$ converges as $\epsilon \rightarrow 0$ to the norm $\|F\|_{0}=\|F\|_{L^{p}\left(\mu_{0}\right)}$ if $F \in C_{k}(B)$.

For more examples of nets of seminorms fulfilling conditions (1)-(3), we refer the reader to 13 .

We say that $F: B \subset \mathbb{R}^{n} \rightarrow \mathbb{R}^{k}$ has a Taylor polynomial of degree $m$ at 0 if there exists $P \in \Pi_{k}^{m}$ such that

$$
\|F-P\|_{\epsilon}^{*}=o\left(\epsilon^{m}\right), \quad \text { as } \epsilon \rightarrow 0 .
$$

It is well known that if a Taylor polynomial exists, it is unique [13, Proposition 3.3]; we denote it by $T_{m}=T_{m}(F)$. We write $F \in t^{m}$ if the function $F$ has the Taylor polynomial of degree $m$ at 0 . Moreover, if $F \in t^{m}$ and $T_{m}(F)=$ $\sum_{|\alpha| \leq m} C_{\alpha} x^{\alpha}$, then the Taylor polynomial of degree $l \leq m$ for $F$ at 0 is given by $T_{l}(F)=\sum_{|\alpha| \leq l} C_{\alpha} x^{\alpha}\left[13\right.$, Proposition 3.5], where $\alpha=\left(\alpha_{1}, \ldots, \alpha_{n}\right) \in \mathbb{R}^{n}$ with $\alpha_{i} \geq 0$ and $|\alpha|=\alpha_{1}+\alpha_{2}+\cdots+\alpha_{n}$. We set $\partial^{\alpha} F(0)$ for the vector $\alpha ! C_{\alpha}$ with $\alpha !=\alpha_{1} ! \alpha_{2} ! \ldots \alpha_{n} !$

The problem of best local approximation with a family of function seminorms $\left\{\|\cdot\|_{\epsilon}\right\}_{\epsilon>0}$ satisfying (1)-(3) was considered in 13 for two types of approximation class $\mathcal{A}$ fulfilling $\Pi_{k}^{m} \subset \mathcal{A} \subset \Pi_{k}^{l}$ and

(c1) $\mathcal{A}^{\epsilon}=\mathcal{A}$, for each $\epsilon>0$, or

(c2) if $P \in \mathcal{A}$ and $T_{m+1}(P)=0$, then $P=0$.

Firstly, the authors studied the asymptotic behavior of a normalized error function as $\epsilon \rightarrow 0$ [13. Theorems 4.2 and 4.5]. Secondly, they showed that there exists the best local approximation to $F$ in 0 and is associated with a Taylor polynomial for $F$ in 0 [13, Theorem 5.1]. In particular, if $\mathcal{A}=\Pi_{k}^{m}$ and $F \in t^{m}$, they proved that $P_{\epsilon} \rightarrow T_{m}(F)$ as $\epsilon \rightarrow 0$ [13. Theorem 3.1].

In this work we generalize the results found in [13], without the restrictions (c1) or (c2) given above. For this, it is essential to study the convergence of the net $\left\{\mathcal{A}^{\epsilon}\right\}$ as $\epsilon \rightarrow 0$.

This paper is organized as follows. In Section 2, we investigate the asymptotic behavior of $\left\{\mathcal{A}^{\epsilon}\right\}$. In Section 3, we study the asymptotic behavior of the error function $\epsilon^{-m-1}\left(F_{\epsilon}-P_{\epsilon}\right)^{\epsilon}$ for a suitable integer, and we show some results about the best local approximation in the origin which generalizes those of [13].

\section{Asymptotic Behavior of the net $\left\{\mathcal{A}^{\epsilon}\right\}$}

In this section, we study the asymptotic behavior of the net $\left\{\mathcal{A}^{\epsilon}\right\}$ given in 1.2 . We begin with the following definition.

Definition 2.1. Let $\mathcal{A} \subset \Pi_{k}^{l}$ be a subspace. We say that $P \in \lim _{\epsilon \rightarrow 0} \mathcal{A}^{\epsilon}$ if there exists a net $\left\{P_{\epsilon}\right\} \subset \mathcal{A}$ such that $\lim _{\epsilon \rightarrow 0}\left\|P-P_{\epsilon}^{\epsilon}\right\|_{0}=0$. We denote $\mathcal{B}=\lim _{\epsilon \rightarrow 0} \mathcal{A}^{\epsilon}$.

Remark 2.2. If $\mathcal{A} \subset \Pi_{k}^{l}$ is a subspace, then the sets $\mathcal{A}^{\epsilon}$ and $\mathcal{B}$ are also subspaces of $\Pi_{k}^{l}$. Furthermore, if $\mathcal{A}^{\epsilon}=\mathcal{A}$, for all $\epsilon>0$, we have that $\mathcal{B}=\mathcal{A}$. 
Next, we show a simple example of $\mathcal{A}^{\epsilon}=\mathcal{A}$.

Example 2.3. Set $n=3$ and $\mathcal{A}=\operatorname{span}\left\{\left(x_{1}, x_{1}+x_{2}+x_{3}, x_{1}^{2}+x_{2}^{2}\right)\right\}$. Then, clearly we obtain $\mathcal{A}^{\epsilon}=\operatorname{span}\left\{\left(\epsilon x_{1}, \epsilon\left(x_{1}+x_{2}+x_{3}\right), \epsilon^{2}\left(x_{1}^{2}+x_{2}^{2}\right)\right)\right\}=\mathcal{A}$.

Proposition 2.4. Let $\mathcal{A}$ be a subspace of polynomials such that $\Pi_{k}^{m} \subset \mathcal{A}$ for some $m \in \mathbb{N} \cup\{0\}$ and $k \in \mathbb{N}$. Then $\Pi_{k}^{m} \subset \mathcal{A}^{\epsilon}$ for all $\epsilon>0$. Moreover, $\Pi_{k}^{m} \subset \mathcal{B}$.

Proof. Set $R_{\alpha, i}(x)=x^{\alpha} e_{i},|\alpha| \leq m, 1 \leq i \leq k$, where $\left\{e_{i}\right\}_{i=1}^{k}$ is the canonical basis of $\mathbb{R}^{k}$. Then

$$
\left\{R_{\alpha, i}:|\alpha| \leq m, 1 \leq i \leq k\right\}
$$

is a basis of the space $\Pi_{k}^{m}$. Since $\mathcal{A}^{\epsilon}$ is a subspace, we have $R_{\alpha, i}=\frac{1}{\epsilon|\alpha|} R_{\alpha, i}^{\epsilon} \in \mathcal{A}^{\epsilon}$, and so $\Pi_{k}^{m} \subset \mathcal{A}^{\epsilon}$, for all $\epsilon>0$. Finally, using the definition of $\mathcal{B}$, we obtain $\Pi_{k}^{m} \subset \mathcal{B}$.

From now on, for any Lebesgue measurable function $F: B \subset \mathbb{R}^{n} \rightarrow \mathbb{R}^{k}$ we denote $T_{-1}(F)=0$.

Proposition 2.5. Let $\mathcal{A}$ be a subspace of $\Pi_{k}^{l}$ and let $0 \leq s+1 \leq l$ be an integer. If $P \in \mathcal{A}$ satisfies $T_{s}(P)=0$ and $T_{s+1}(P) \neq 0$, then $T_{s+1}(P) \in \mathcal{B}$.

Proof. For each $\epsilon>0$ we define $Q_{\epsilon}=\frac{P}{\epsilon^{s+1}} \in \mathcal{A}$. Since $T_{s}(P)=0$, it follows that $\left\|T_{s+1}(P)-Q_{\epsilon}^{\epsilon}\right\|_{0}=\frac{\left\|\left(T_{s+1}(P)-P\right)^{\epsilon}\right\|_{0}}{\epsilon^{s+1}}$. So $\left\|T_{s+1}(P)-Q_{\epsilon}^{\epsilon}\right\|_{0}=o(1)$ as $\epsilon \rightarrow 0$, and thus $T_{s+1}(P) \in \mathcal{B}$.

The following sets will be needed throughout the paper. Let $\mathcal{A}$ be a non-zero subspace of $\Pi_{k}^{l}$. We define

$$
A_{-1}:=\mathcal{A} \quad \text { and } \quad A_{j}:=\left\{P \in \mathcal{A}: T_{j}(P)=0\right\}, \text { for } 0 \leq j \leq l .
$$

We note that

$$
A_{j} \subset A_{i}, \quad \text { whenever } i<j .
$$

Since $A_{l} \subset\left\{P \in \Pi_{k}^{l}: T_{l}(P)=0\right\}=\{0\}$, we have

$$
\left\{j: 0 \leq j \leq l \text { and } A_{j} \neq \mathcal{A}\right\} \neq \emptyset \quad \text { and } \quad\left\{j: 0 \leq j \leq l \text { and } A_{j}=\{0\}\right\} \neq \emptyset .
$$

Set

$$
s_{0}=\min \left\{j: 0 \leq j \leq l \text { and } A_{j} \neq \mathcal{A}\right\}
$$

and

$$
r_{0}=\min \left\{j: 0 \leq j \leq l \text { and } A_{j}=\{0\}\right\} .
$$

It is easy to see that $0 \leq s_{0} \leq r_{0} \leq l$, and

$$
s_{0}, r_{0} \in\left\{j: s_{0} \leq j \leq r_{0} \text { and } A_{j} \subsetneq A_{j-1}\right\}=: J .
$$

We can now formulate our main result which describes the limit set $\mathcal{B}$.

Theorem 2.6. Let $\mathcal{A}$ be a non-zero subspace of $\Pi_{k}^{l}$. Then $\mathcal{B}$ is a subspace of $\Pi_{k}^{r_{0}}$ isomorphic to $\mathcal{A}$. Furthermore, under the above notation the following holds:

(a) if $s_{0}<r_{0}$ and $J \backslash\left\{r_{0}\right\}=\left\{s_{0}, \ldots, s_{N}\right\}$ with $s_{i}<s_{i+1}$ for $N>0$, then $\mathcal{B}=$ $T_{r_{0}}\left(A_{s_{N}}\right) \oplus T_{s_{N}}\left(S_{s_{N}}\right) \oplus T_{s_{N-1}}\left(S_{s_{N-1}}\right) \oplus \cdots \oplus T_{s_{0}}\left(S_{s_{0}}\right)$, where $A_{s_{i}} \oplus S_{s_{i}}=A_{s_{i-1}}$, $0 \leq i \leq N$; 
(b) if $s_{0}=r_{0}$, then $\mathcal{B}=T_{r_{0}}(\mathcal{A})$.

Proof. (a) Assume $s_{0}<r_{0}$. Since every subspace of $A_{s_{i-1}}, 0 \leq i \leq N$, has a complement, there exists a subspace $S_{s_{i}} \subset A_{s_{i-1}}$ such that

$$
A_{s_{i}} \oplus S_{s_{i}}=A_{s_{i-1}}, \quad 0 \leq i \leq N .
$$

In consequence,

$$
\mathcal{A}=A_{s_{N}} \oplus S_{s_{N}} \oplus S_{s_{N-1}} \oplus \cdots \oplus S_{s_{0}} .
$$

As $S_{s_{i}} \subset A_{s_{i-1}}, 0 \leq i \leq N$, and $A_{r_{0}-1}=A_{s_{N}}$, we obtain

$$
Q(x)= \begin{cases}\sum_{|\alpha| \geq s_{i}} \frac{\partial^{\alpha} Q(0)}{\alpha !} x^{\alpha}, & \text { if } Q \in S_{s_{i}}, 0 \leq i \leq N \\ \sum_{|\alpha| \geq s_{N+1}} \frac{\partial^{\alpha} Q(0)}{\alpha !} x^{\alpha}, & \text { if } Q \in A_{s_{N}},\end{cases}
$$

where $s_{N+1}=r_{0}$. Let $T_{i}: S_{s_{i}} \rightarrow \Pi_{k}^{s_{i}}$ be a linear operator defined by $T_{i}(P)=$ $T_{s_{i}}(P), 0 \leq i \leq N$, and $T_{N+1}: \mathcal{A} \rightarrow \Pi_{k}^{s_{N+1}}$ be the linear operator given by $T_{N+1}(P)=T_{s_{N+1}}(P)$. We claim that

(i) $T_{i}$ is an injective operator, $0 \leq i \leq N+1$.

(ii) $T_{s_{N+1}}\left(A_{s_{N}}\right) \cap \sum_{i=0}^{N} T_{s_{i}}\left(S_{s_{i}}\right)=\{0\}$.

(iii) If $N>0$ then $T_{s_{l}}\left(S_{s_{l}}\right) \cap\left(T_{s_{N+1}}\left(A_{s_{N}}\right)+\sum_{i=0, i \neq l}^{N} T_{s_{i}}\left(S_{s_{i}}\right)\right)=\{0\}$ whenever $l \neq i$.

Indeed, let $0 \leq i \leq N$. If $T_{s_{i}}(P)=T_{s_{i}}(Q)$ for some $P, Q \in S_{s_{i}}$, then $P-Q \in$ $A_{s_{i}} \cap S_{s_{i}}$. So 2.3 implies that $P=Q$. On the other hand, if $T_{s_{N+1}}(P)=T_{s_{N+1}}(Q)$ with $P, Q \in \mathcal{A}$, then $P-Q \in A_{s_{N+1}}=\{0\}$, which proves (i). To prove (ii) we consider $Q_{N+1} \in A_{s_{N}}$ and $Q_{i} \in S_{s_{i}}$ such that $P=T_{s_{N+1}}\left(Q_{N+1}\right)=\sum_{i=0}^{N} T_{s_{i}}\left(Q_{i}\right)$. From 2.5) we see that

$$
T_{s_{N+1}}\left(Q_{N+1}\right)(x)=\sum_{|\alpha|=s_{N+1}} \frac{\partial^{\alpha} Q_{N}(0)}{\alpha !} x^{\alpha} \quad \text { and } \quad \sum_{i=0}^{N} T_{s_{i}}\left(Q_{i}\right) \in \Pi_{k}^{s_{N}} .
$$

Therefore $P=0$. Now, let $Q_{N+1} \in A_{s_{N}}$ and $Q_{i} \in S_{s_{i}}$ be such that

$$
P=T_{s_{l}}\left(Q_{l}\right)=T_{s_{N+1}}\left(Q_{N+1}\right)+\sum_{i=0, i \neq l}^{N} T_{s_{i}}\left(Q_{i}\right) .
$$

From 2.5 it follows that

$$
T_{s_{i}}\left(Q_{i}\right)=\sum_{|\alpha|=s_{i}} \frac{\partial^{\alpha} Q_{i}(0)}{\alpha !} x^{\alpha}, \quad 0 \leq i \leq N .
$$

According to 2.6 and 2.7 we have $P=0$, and (iii) is proved. Using (i)-(iii), we deduce that the subspace

$$
T_{s_{N+1}}\left(A_{s_{N}}\right)+T_{s_{N}}\left(S_{s_{N}}\right)+T_{s_{N-1}}\left(S_{s_{N-1}}\right)+\cdots+T_{s_{0}}\left(S_{s_{0}}\right)
$$

is a direct sum isomorphic to $\mathcal{A}$. The proof concludes by proving

$$
\mathcal{B}=T_{s_{N+1}}\left(A_{s_{N}}\right) \oplus T_{s_{N}}\left(S_{s_{N}}\right) \oplus T_{s_{N-1}}\left(S_{s_{N-1}}\right) \oplus \cdots \oplus T_{s_{0}}\left(S_{s_{0}}\right) .
$$


We observe that if $P \in S_{s_{i}} \backslash\{0\}$, then $T_{s_{i}}(P) \neq 0$ and $T_{s_{i}-1}(P)=0$ by 2.3 . So, Proposition 2.5 implies that $T_{s_{i}}(P) \in \mathcal{B}$. On the other hand, if $P \in A_{s_{N}} \backslash\{0\}$, we get $T_{s_{N}}(P)=0$. Moreover, we have $T_{s_{N+1}}(P) \neq 0$. In fact, on the contrary, we see that $P \in A_{s_{N+1}}=\{0\}$. Proposition 2.5 now gives $T_{s_{N+1}}(P) \in \mathcal{B}$. Therefore,

$$
T_{s_{N+1}}\left(A_{s_{N}}\right) \oplus T_{s_{N}}\left(S_{s_{N}}\right) \oplus T_{s_{N-1}}\left(S_{s_{N-1}}\right) \oplus \cdots \oplus T_{s_{0}}\left(S_{s_{0}}\right) \subset \mathcal{B} .
$$

On the other hand, if $P \in \mathcal{B}$, there exists $\left\{P_{\epsilon}\right\} \subset \mathcal{A}$ such that

$$
\lim _{\epsilon \rightarrow 0}\left\|P-P_{\epsilon}^{\epsilon}\right\|_{0}=0 \text {. }
$$

Let $d_{N+1}=\operatorname{dim}\left(A_{s_{N}}\right)$ and $d_{i}=\operatorname{dim}\left(S_{s_{i}}\right), 0 \leq i \leq N$. We take $\left\{v_{l}\right\}_{l=1}^{d_{N+1}}$ and $\left\{w_{i r}\right\}_{r=1}^{d_{i}}$ bases of $A_{s_{N}}$ and $S_{s_{i}}$, respectively. It is easy to check that for each $0<\epsilon \leq 1,\left\{\epsilon^{-s_{N+1}} v_{l}\right\}_{l=1}^{d_{N+1}}$ is a basis of $A_{s_{N}}$ and $\left\{\epsilon^{-s_{i}} w_{i r}\right\}_{r=1}^{d_{i}}$ is a basis of $S_{s_{i}}$, $0 \leq i \leq N$. According to 2.4, we have that there exist real numbers $D_{l, \epsilon}$ and $C_{i, r, \epsilon}$ such that

$$
P_{\epsilon}=\sum_{l=1}^{d_{N+1}} \epsilon^{-s_{N+1}} D_{l, \epsilon} v_{l}+\sum_{i=0}^{N} \sum_{r=1}^{d_{i}} \epsilon^{-s_{i}} C_{i, r, \epsilon} w_{i r}
$$

From 2.5 it follows that

$$
v_{l}(x)=\sum_{|\alpha| \geq s_{N+1}} \frac{\partial^{\alpha} v_{l}(0)}{\alpha !} x^{\alpha} \quad \text { and } \quad w_{i r}(x)=\sum_{|\alpha| \geq s_{i}} \frac{\partial^{\alpha} w_{i r}(0)}{\alpha !} x^{\alpha} .
$$

So,

$$
\begin{aligned}
P_{\epsilon}^{\epsilon}(x)= & \sum_{l=1}^{d_{N+1}} D_{l, \epsilon} \epsilon^{-s_{N+1}} v_{l}^{\epsilon}(x)+\sum_{i=0}^{N} \sum_{r=1}^{d_{i}} C_{i, r, \epsilon} \epsilon^{-s_{i}} w_{i r}^{\epsilon}(x) \\
= & \sum_{l=1}^{d_{N+1}} D_{l, \epsilon} \sum_{|\alpha|=s_{N+1}} \frac{\partial^{\alpha} v_{l}(0)}{\alpha !} x^{\alpha}+\sum_{l=1}^{d_{N+1}} D_{l, \epsilon} \sum_{|\alpha|>s_{N+1}} \epsilon^{|\alpha|-s_{N+1}} \frac{\partial^{\alpha} v_{l}(0)}{\alpha !} x^{\alpha} \\
& +\sum_{i=0}^{N} \sum_{r=1}^{d_{i}} C_{i, r, \epsilon} \sum_{|\alpha|=s_{i}} \frac{\partial^{\alpha} w_{i r}(0)}{\alpha !} x^{\alpha}+\sum_{i=0}^{N} \sum_{r=1}^{d_{i}} C_{i, r, \epsilon} \sum_{|\alpha|>s_{i}} \epsilon^{|\alpha|-s_{i}} \frac{\partial^{\alpha} w_{i r}(0)}{\alpha !} x^{\alpha} .
\end{aligned}
$$

Consequently

$$
\begin{aligned}
T_{s_{j}}\left(P_{\epsilon}^{\epsilon}\right)(x)= & \sum_{i=0}^{j} \sum_{r=1}^{d_{i}} C_{i, r, \epsilon} \sum_{|\alpha|=s_{i}} \frac{\partial^{\alpha} w_{i r}(0)}{\alpha !} x^{\alpha} \\
& +\sum_{i=0}^{j-1} \sum_{r=1}^{d_{i}} C_{i, r, \epsilon} \sum_{s_{i}<|\alpha| \leq s_{j}} \epsilon^{|\alpha|-s_{i}} \frac{\partial^{\alpha} w_{i r}(0)}{\alpha !} x^{\alpha}
\end{aligned}
$$


if $0 \leq j \leq N$, and

$$
\begin{aligned}
T_{s_{N+1}}\left(P_{\epsilon}^{\epsilon}\right)(x)= & \sum_{l=1}^{d_{N+1}} D_{l, \epsilon} \sum_{|\alpha|=s_{N+1}} \frac{\partial^{\alpha} v_{l}(0)}{\alpha !} x^{\alpha}+\sum_{i=0}^{N} \sum_{r=1}^{d_{i}} C_{i, r, \epsilon} \sum_{|\alpha|=s_{i}} \frac{\partial^{\alpha} w_{i r}(0)}{\alpha !} x^{\alpha} \\
& +\sum_{i=0}^{N} \sum_{r=1}^{d_{i}} C_{i, r, \epsilon} \sum_{s_{i}<|\alpha| \leq s_{N+1}} \epsilon^{|\alpha|-s_{i}} \frac{\partial^{\alpha} w_{i r}(0)}{\alpha !} x^{\alpha} .
\end{aligned}
$$

From $(2.9)$ it follows that

$$
T_{s_{N+1}}\left(v_{\ell}\right)(x)=\sum_{|\alpha|=s_{N+1}} \frac{\partial^{\alpha} v_{\ell}(0)}{\alpha !} x^{\alpha} \quad \text { and } \quad T_{s_{j}}\left(w_{j . r}\right)(x)=\sum_{|\alpha|=s_{j}} \frac{\partial^{\alpha} w_{j, r}(0)}{\alpha !} x^{\alpha} .
$$

Thus, a straightforward computation yields

$$
\begin{gathered}
T_{s_{0}}\left(P_{\epsilon}^{\epsilon}\right)(x)=\sum_{r=1}^{d_{0}} C_{0, r, \epsilon} T_{s_{0}}\left(w_{0, r}\right)(x) \\
T_{s_{j}}\left(P_{\epsilon}^{\epsilon}\right)(x)=T_{s_{j-1}}\left(P_{\epsilon}^{\epsilon}\right)(x)+\sum_{i=0}^{j-1} \sum_{r=1}^{d_{i}} C_{i, r, \epsilon} \sum_{s_{j-1}<|\alpha| \leq s_{j}} \epsilon^{|\alpha|-s_{i}} \frac{\partial^{\alpha} w_{i, r}(0)}{\alpha !} x^{\alpha} \\
+\sum_{r=1}^{d_{j}} C_{j, r, \epsilon} T_{s_{j}}\left(w_{j, r}\right)(x)
\end{gathered}
$$

if $1 \leq j \leq N$, and

$$
\begin{aligned}
T_{s_{N+1}}\left(P_{\epsilon}^{\epsilon}\right)(x)= & T_{s_{N}}\left(P_{\epsilon}^{\epsilon}\right)(x)+\sum_{l=1}^{d_{N+1}} D_{l, \epsilon} T_{s_{N+1}}\left(v_{\ell}\right)(x) \\
& +\sum_{i=0}^{N} \sum_{r=1}^{d_{i}} C_{i, r, \epsilon} \sum_{s_{N}<|\alpha| \leq s_{N+1}} \epsilon^{|\alpha|-s_{i}} \frac{\partial^{\alpha} w_{i, r}(0)}{\alpha !} x^{\alpha} .
\end{aligned}
$$

From 2.8 and 2.10, we deduce that $T_{s_{0}}\left(P_{\epsilon}^{\epsilon}\right)(x)=\sum_{r=1}^{d_{0}} C_{0, r, \epsilon} T_{s_{0}}\left(w_{0, r}\right)(x)$ is convergent as $\epsilon \rightarrow 0$. Since $\left\{T_{s_{0}}\left(w_{0, r}\right)\right\}_{r=1}^{d_{0}}$ is a basis of $T_{s_{0}}\left(S_{s_{0}}\right)$, there are real numbers $C_{0, r}, 1 \leq r \leq d_{0}$, such that $C_{0, r, \epsilon} \rightarrow C_{0, r}$ as $\epsilon \rightarrow 0$. According to 2.8 and 2.11) it follows that $\sum_{r=1}^{d_{1}} C_{1, r, \epsilon} T_{s_{1}}\left(w_{1, r}\right)(x)$ is convergent as $\epsilon \rightarrow 0$. Hence, there are real numbers $C_{1, r}, 1 \leq r \leq d_{1}$, such that $C_{1, r, \epsilon} \rightarrow C_{1, r}$ as $\epsilon \rightarrow 0$, because $\left\{T_{s_{1}}\left(w_{1 r}\right)\right\}_{r=1}^{d_{1}}$ is a basis of $T_{s_{1}}\left(S_{s_{1}}\right)$. Similarly, as $\left\{T_{s_{N+1}}\left(v_{l}\right)\right\}_{l=1}^{a}$ is a basis of $T_{s_{N+1}}\left(A_{s_{N}}\right)$ and $\left\{T_{s_{i}}\left(w_{i r}\right)\right\}_{r=1}^{d_{i}}$ is a basis of $T_{s_{i}}\left(S_{s_{i}}\right), 0 \leq i \leq N, 2.8$ and 2.10 (2.12) show that there are real numbers $D_{l}$ and $C_{i, r}$ such that $D_{l, \epsilon}, \rightarrow D_{l}$ and $C_{i, r, \epsilon} \rightarrow C_{i, r}$ as $\epsilon \rightarrow 0$. In consequence,

$$
P=\sum_{l=1}^{a} D_{l} T_{s_{N+1}}\left(v_{l}\right)+\sum_{i=0}^{N}\left(\sum_{r=1}^{d_{i}} C_{i, r} T_{s_{i}}\left(w_{i r}\right)\right),
$$

and so $P \in T_{s_{N+1}}\left(A_{s_{N}}\right) \oplus T_{s_{N}}\left(S_{s_{N}}\right) \oplus T_{s_{N-1}}\left(S_{s_{N-1}}\right) \oplus \cdots \oplus T_{s_{0}}\left(S_{s_{0}}\right)$. 
(b) Now assume $s_{0}=r_{0}$, i.e., $A_{s_{0}}=\{0\}$. Then $\mathcal{A}$ has the form 2.4 with $N=0$, $A_{s_{0}}=\{0\}$ and $S_{s_{0}}=\mathcal{A}$. An analysis similar to the proof of (a) shows that $T_{r_{0}}$ is an isomorphism and $\mathcal{B}=T_{s_{0}}\left(S_{s_{0}}\right)=T_{r_{0}}(\mathcal{A})$.

The following corollary follows immediately from the proof of Theorem 2.6

Corollary 2.7. Let $\mathcal{A}$ be a non-zero subspace of $\Pi_{k}^{l}$. Then $\lim _{n \rightarrow \infty} \mathcal{A}^{\epsilon_{n}}=\mathcal{B}$ for any sequence $\left\{\epsilon_{n}\right\}$ of the net $\epsilon \downarrow 0$.

Remark 2.8. $\mathcal{B}$ is isomorphic to $T_{r_{0}}(\mathcal{A})$.

Corollary 2.9. Let $s \geq m+1$ and let $\mathcal{A}=\Pi_{k}^{m} \oplus A_{s-1}$ be such that $A_{s}=\{0\}$. Then $\mathcal{B}=\Pi_{k}^{m} \oplus T_{s}\left(A_{s-1}\right)$ and the linear operator $T: \mathcal{A} \rightarrow \Pi_{k}^{s}$ given by $T(P)=T_{s}(P)$ defines an isomorphism between $\mathcal{A}$ and $\mathcal{B}$.

Proof. We first claim that $T$ is an injective operator. Indeed, if $T(P)=T(Q)$ for $P, Q \in \mathcal{A}$, then $T_{s}(P-Q)=0$ and so $P-Q \in A_{s}$. Since $A_{s}=\{0\}$, we have $P=Q$.

Since $\mathcal{A}$ is isomorphic to $T(\mathcal{A})$, the proof concludes by proving $\mathcal{B}=\Pi_{k}^{m} \oplus$ $T_{s}\left(A_{s-1}\right)=T_{s}(\mathcal{A})$.

Let $A_{j}$ be the sets defined in 2.2 . Since

$$
\{0\}=A_{s} \subsetneq A_{s-1}=\cdots=A_{m} \subsetneq A_{m-1} \subsetneq \cdots \subsetneq A_{0} \subsetneq \mathcal{A},
$$

then $\mathcal{A}=A_{s-1} \oplus B_{m} \oplus B_{m-1} \oplus \cdots \oplus B_{0}$, where $A_{i} \oplus B_{i}=A_{i-1}, 0 \leq i \leq m$. Therefore $\Pi_{k}^{m}$ is isomorphic to $B_{m} \oplus \cdots \oplus B_{0}$. On the other hand, since $s_{0}=0$, $r_{0}=s$ and $J \backslash\left\{r_{0}\right\}=\{0,1, \ldots, m\}$, by Proposition 2.6 (a),

$$
\mathcal{B}=T_{s}\left(A_{s-1}\right) \oplus T_{m}\left(B_{m}\right) \oplus \cdots \oplus T_{0}\left(B_{0}\right) .
$$

From the proof of Theorem 2.6, we obtain that $B_{m} \oplus \cdots \oplus B_{0}$ is isomorphic to $T_{m}\left(B_{m}\right) \oplus \cdots \oplus T_{0}\left(B_{0}\right)$, and consequently $\Pi_{k}^{m}$ is isomorphic to $T_{m}\left(B_{m}\right) \oplus \cdots \oplus$ $T_{0}\left(B_{0}\right) \subset \Pi_{k}^{m}$. Hence, $T_{m}\left(B_{m}\right) \oplus \cdots \oplus T_{0}\left(B_{0}\right)=\Pi_{k}^{m}$ and so $\mathcal{B}=T_{s}\left(A_{s-1}\right) \oplus \Pi_{k}^{m}=$ $T_{s}\left(A_{s-1}\right) \oplus T_{s}\left(\Pi_{k}^{m}\right)=T_{s}(\mathcal{A})$.

\section{An application to Best local approximation}

Let $\left\{P_{\epsilon}\right\}$ be a net of best approximants to $F$ from $\mathcal{A}$ with respect to $\|\cdot\|_{\epsilon}^{*}$, and let $E_{\epsilon}$ be the error function

$$
E_{\epsilon}(F)=\frac{F^{\epsilon}-P_{\epsilon}^{\epsilon}}{\epsilon^{m+1}}
$$

If $F \in t^{m+1}$, then

$$
F^{\epsilon}=T_{m+1}^{\epsilon}+\epsilon^{m+1} R_{m+1}^{\epsilon},
$$

where $R_{m+1}=\frac{F-T_{m+1}}{\epsilon^{m+1}},\left\|R_{m+1}^{\epsilon}\right\|_{\epsilon}=o(1)$, and $T_{m+1}$ is the Taylor polynomial of $F$ of degree $m+1$ at 0 . Moreover,

$$
\lambda P_{\epsilon}^{\epsilon} \in \mathcal{P}_{\mathcal{A}^{\epsilon}, \epsilon}\left(\lambda F^{\epsilon}\right) \quad \text { and } \quad P^{\epsilon}+P_{\epsilon}^{\epsilon} \in \mathcal{P}_{\mathcal{A}^{\epsilon}, \epsilon}\left((P+F)^{\epsilon}\right), \quad \text { for } P \in \mathcal{A} .
$$

The following proposition may be proved in much the same way as [13. Proposition 4.1]. However, we repeat the proof for completeness. 
Proposition 3.1. Let $\mathcal{A}$ be a non-zero subspace of $\Pi_{k}^{l}$ with $l>m$, and let $\left\{P_{\epsilon}\right\}$ be a net of best approximants of $F$ from $\mathcal{A}$ with respect to $\|\cdot\|_{\epsilon}^{*}$. If $F \in t^{m+1}, T_{m} \in \mathcal{A}$ and $\phi_{m+1}=T_{m+1}-T_{m}$, then

$$
E_{\epsilon}(F)=\phi_{m+1}+R_{m+1}^{\epsilon}-\mathcal{P}_{\mathcal{A}^{\epsilon}, \epsilon}\left(\phi_{m+1}+R_{m+1}^{\epsilon}\right),
$$

where $\left\|R_{m+1}^{\epsilon}\right\|_{\epsilon}=o(1)$ as $\epsilon \rightarrow 0$.

Proof. Since $R_{m+1}^{\epsilon}=\frac{F^{\epsilon}-T_{m+1}^{\epsilon}}{\epsilon^{m+1}}$, then

$$
\begin{aligned}
\phi_{m+1}+R_{m+1}^{\epsilon} & =T_{m+1}-T_{m}+\frac{F^{\epsilon}-T_{m+1}^{\epsilon}}{\epsilon^{m+1}}=\frac{T_{m+1}^{\epsilon}-T_{m}^{\epsilon}}{\epsilon^{m+1}}+\frac{F^{\epsilon}-T_{m+1}^{\epsilon}}{\epsilon^{m+1}} \\
& =\frac{F^{\epsilon}-T_{m}^{\epsilon}}{\epsilon^{m+1}} .
\end{aligned}
$$

As $T_{m} \in \mathcal{A}$, we have

$$
\begin{aligned}
\phi_{m+1}+R_{m+1}^{\epsilon}-\mathcal{P}_{\mathcal{A}^{\epsilon}, \epsilon}\left(\phi_{m+1}+R_{m+1}^{\epsilon}\right) & =\frac{F^{\epsilon}-T_{m}^{\epsilon}}{\epsilon^{m+1}}-P_{\mathcal{A}^{\epsilon}, \epsilon}\left(\frac{F^{\epsilon}-T_{m}^{\epsilon}}{\epsilon^{m+1}}\right) \\
& =\frac{F^{\epsilon}-P_{\epsilon}^{\epsilon}}{\epsilon^{m+1}}=E_{\epsilon}(F) .
\end{aligned}
$$

Next, we give a new result about the asymptotic behavior of the error without the conditions $(\mathrm{c} 1)$ or $(\mathrm{c} 2)$, which generalizes Theorems 4.2 and 4.5 of [13].

Theorem 3.2. Let $\mathcal{A}$ be a non-zero subspace of $\Pi_{k}^{l}$ with $l>m$. If $F \in t^{m+1}$, $T_{m} \in \mathcal{A}$ and $\phi_{m+1}=T_{m+1}-T_{m}$, then

$$
\left\|E_{\epsilon}(F)\right\|_{\epsilon} \rightarrow \inf _{P \in \mathcal{B}}\left\|\phi_{m+1}-P\right\|_{0}, \quad \text { as } \epsilon \rightarrow 0 .
$$

Proof. By Proposition 3.1

$$
E_{\epsilon}(F)=\phi_{m+1}+R_{m+1}^{\epsilon}-\mathcal{P}_{\mathcal{A}^{\epsilon}, \epsilon}\left(\phi_{m+1}+R_{m+1}^{\epsilon}\right),
$$

where $\left\|R_{m+1}^{\epsilon}\right\|_{\epsilon}=o(1)$ as $\epsilon \rightarrow 0$. We first prove

$$
\varlimsup_{\epsilon \rightarrow 0}\left\|E_{\epsilon}(F)\right\|_{\epsilon} \leq \inf _{P \in B}\left\|\phi_{m+1}-P\right\|_{0} .
$$

In fact, let $P \in \mathcal{B}$. By the definition of $\mathcal{B}$, there exists a net $\left\{Q_{\epsilon}\right\} \subset \mathcal{A}$ such that $\left\|P-Q_{\epsilon}^{\epsilon}\right\|_{0} \rightarrow 0$, as $\epsilon \rightarrow 0$. In consequence, $\left\|P-Q_{\epsilon}^{\epsilon}\right\|_{\epsilon}=o(1)$, as $\epsilon \rightarrow 0$, by (1.3). Since $Q_{\epsilon}^{\epsilon} \in \mathcal{A}^{\epsilon}$ and $\left\|R_{m+1}^{\epsilon}\right\|_{\epsilon}=o(1)$, from 3.1 we obtain

$$
\left\|E_{\epsilon}(F)\right\|_{\epsilon} \leq\left\|\phi_{m+1}+R_{m+1}^{\epsilon}-Q_{\epsilon}^{\epsilon}\right\|_{\epsilon} \leq\left\|\phi_{m+1}-Q_{\epsilon}^{\epsilon}\right\|_{\epsilon}+o(1), \quad \text { as } \epsilon \rightarrow 0 .
$$

By Property (3), $\left\|\phi_{m+1}-P\right\|_{\epsilon} \rightarrow\left\|\phi_{m+1}-P\right\|_{0}$, as $\epsilon \rightarrow 0$. Hence, using the triangle inequality we have

$$
\begin{aligned}
& \left|\left\|\phi_{m+1}-Q_{\epsilon}^{\epsilon}\right\|_{\epsilon}-\left\|\phi_{m+1}-P\right\|_{0}\right| \leq\left|\left\|\phi_{m+1}-Q_{\epsilon}^{\epsilon}\right\|_{\epsilon}-\left\|\phi_{m+1}-P\right\|_{\epsilon}\right| \\
& +\left|\left\|\phi_{m+1}-P\right\|_{\epsilon}-\left\|\phi_{m+1}-P\right\|_{0}\right| \\
& \quad \leq\left\|P-Q_{\epsilon}^{\epsilon}\right\|_{\epsilon}+\left|\left\|\phi_{m+1}-P\right\|_{\epsilon}-\left\|\phi_{m+1}-P\right\|_{0}\right|=o(1)
\end{aligned}
$$

as $\epsilon \rightarrow 0$. Now, according to 3.3 we get 3.2 . 
The proof finishes by observing that

$$
\varliminf_{\epsilon \rightarrow 0}\left\|E_{\epsilon}(F)\right\|_{\epsilon} \geq \inf _{P \in \mathcal{B}}\left\|\phi_{m+1}-P\right\|_{0} .
$$

Let $\epsilon \downarrow 0$ be a sequence such that $\lim _{\epsilon \rightarrow 0}\left\|E_{\epsilon}(F)\right\|_{\epsilon}=\underline{\lim }_{\epsilon \rightarrow 0}\left\|E_{\epsilon}(F)\right\|_{\epsilon}$. We consider $P_{\epsilon}^{\epsilon} \in \mathcal{P}_{\mathcal{A}^{\epsilon}, \epsilon}\left(\phi_{m+1}+R_{m+1}^{\epsilon}\right)$. We claim that there exist constants $M, \epsilon_{0}>0$ such that

$$
\left\|P_{\epsilon}^{\epsilon}\right\|_{0} \leq M, \quad 0<\epsilon \leq \epsilon_{0}
$$

Indeed, as $0 \in \mathcal{A}^{\epsilon}$ we get

$$
\begin{aligned}
\left\|P_{\epsilon}^{\epsilon}\right\|_{\epsilon} & \leq\left\|P_{\epsilon}^{\epsilon}-\left(\phi_{m+1}+R_{m+1}^{\epsilon}\right)\right\|_{\epsilon}+\left\|\phi_{m+1}+R_{m+1}^{\epsilon}\right\|_{\epsilon} \\
& \leq 2\left\|\phi_{m+1}+R_{m+1}^{\epsilon_{n}}\right\|_{\epsilon} \\
& \leq 2\left\|\phi_{m+1}\right\|_{\epsilon}+2\left\|R_{m+1}^{\epsilon}\right\|_{\epsilon},
\end{aligned}
$$

for $0<\epsilon \leq 1$. By Proposition 3.1 and Property (3), we see that $2\left\|\phi_{m+1}\right\|_{\epsilon}+$ $2\left\|R_{m+1}^{\epsilon}\right\|_{\epsilon} \rightarrow 2\left\|\phi_{m+1}\right\|_{0}$, as $\epsilon \rightarrow 0$. So, from (1.3) and (3.6), we obtain 3.5).

In consequence, there exists a subsequence of $\left\{P_{\epsilon}^{\epsilon}\right\}$, which is denoted in the same way, and $P_{0} \in \Pi_{k}^{l}$ such that $P_{\epsilon}^{\epsilon} \rightarrow P$ uniformly on $B$, as $\epsilon \rightarrow 0$. Since $\left|\left\|\phi_{m+1}-P_{\epsilon}^{\epsilon}\right\|_{\epsilon}-\left\|\phi_{m+1}-P\right\|_{0}\right| \leq\left|\left\|\phi_{m+1}-P_{\epsilon}^{\epsilon}\right\|_{\epsilon}-\left\|\phi_{m+1}-P\right\|_{\epsilon}\right|+\mid\left\|\phi_{m+1}-P\right\|_{\epsilon}-$ $\left\|\phi_{m+1}-P\right\|_{0}\left|\leq\left\|P-P_{\epsilon}^{\epsilon}\right\|_{\epsilon}+\right|\left\|\phi_{m+1}-P\right\|_{\epsilon}-\left\|\phi_{m+1}-P\right\|_{0} \mid$, using Property (3) we get

$$
\left\|\phi_{m+1}-P\right\|_{0}=\left\|\phi_{m+1}-P_{\epsilon}^{\epsilon}\right\|_{\epsilon}+o(1), \quad \text { as } \epsilon \rightarrow 0 .
$$

We observe that $P \in B$ by Corollary 2.7 Therefore, by Proposition 3.1

$$
\begin{aligned}
\inf _{Q \in \mathcal{B}}\left\|\phi_{m+1}-Q\right\|_{0} & \leq\left\|\phi_{m+1}-P\right\|_{0}=\left\|\phi_{m+1}-P_{\epsilon}^{\epsilon}\right\|_{\epsilon}+o(1) \\
& \leq\left\|\phi_{m+1}+R_{m+1}^{\epsilon}-P_{\epsilon}^{\epsilon}\right\|_{\epsilon}+\left\|R_{m+1}^{\epsilon}\right\|_{\epsilon} \\
& =\left\|E_{\epsilon}(F)\right\|_{\epsilon}+\left\|R_{m+1}^{\epsilon}\right\|_{\epsilon} .
\end{aligned}
$$

So, $\inf _{Q \in \mathcal{B}}\left\|\phi_{m+1}-Q\right\|_{0} \leq \lim _{\epsilon \rightarrow 0}\left(\left\|E_{\epsilon}(F)\right\|_{\epsilon}+\left\|R_{m+1}^{\epsilon}\right\|_{\epsilon}\right)=\underline{\lim }_{\epsilon \rightarrow 0}\left\|E_{\epsilon}(F)\right\|_{\epsilon}$, and 3.4 is proved.

The following result provides us with a useful and important property for a net of best approximants to $F$ from $\mathcal{A}$.

Theorem 3.3. Let $\mathcal{A}$ be a non-zero subspace of $\Pi_{k}^{l}$ with $l>m$, and let $\left\{P_{\epsilon}\right\}$ be a net of best approximants of $F$ from $\mathcal{A}$ with respect to $\|\cdot\|_{\epsilon}^{*}$. Assume $F \in t^{m+1}, T_{m} \in \mathcal{A}$ and $\phi_{m+1}=T_{m+1}-T_{m}$. If $\mathcal{C}$ is the cluster point set of the net $\left\{\frac{\left(P_{\epsilon}-T_{m}\right)^{\epsilon}}{\epsilon^{m+1}}\right\}$, as $\epsilon \rightarrow 0$, then $\mathcal{C} \neq \emptyset$. Moreover, each polynomial in $\mathcal{C}$ is a solution of the minimization problem

$$
\min _{P \in \mathcal{B}}\left\|\phi_{m+1}-P\right\|_{0}
$$


Proof. We observe that

$$
\begin{aligned}
E_{\epsilon}(F) & =\frac{\left(F-P_{\epsilon}\right)^{\epsilon}}{\epsilon^{m+1}}=\frac{\left(T_{m+1}-T_{m}\right)^{\epsilon}+\left(F-T_{m+1}\right)^{\epsilon}-\left(P_{\epsilon}-T_{m}\right)^{\epsilon}}{\epsilon^{m+1}} \\
& =\frac{\phi_{m+1}^{\epsilon}-\left(P_{\epsilon}-T_{m}\right)^{\epsilon}}{\epsilon^{m+1}}+\frac{\left(F-T_{m+1}\right)^{\epsilon}}{\epsilon^{m+1}} \\
& =\phi_{m+1}-\frac{\left(P_{\epsilon}-T_{m}\right)^{\epsilon}}{\epsilon^{m+1}}+\frac{\left(F-T_{m+1}\right)^{\epsilon}}{\epsilon^{m+1}} .
\end{aligned}
$$

Then

$$
\begin{aligned}
\| \phi_{m+1}-\frac{\left(P_{\epsilon}-T_{m}\right)^{\epsilon}}{\epsilon^{m+1}} & \|_{\epsilon}-\frac{\left\|\left(F-T_{m+1}\right)^{\epsilon}\right\|_{\epsilon}}{\epsilon^{m+1}} \\
& \leq\left\|E_{\epsilon}(F)\right\|_{\epsilon} \leq\left\|\phi_{m+1}-\frac{\left(P_{\epsilon}-T_{m}\right)^{\epsilon}}{\epsilon^{m+1}}\right\|_{\epsilon}+\frac{\left\|\left(F-T_{m+1}\right)^{\epsilon}\right\|_{\epsilon}}{\epsilon^{m+1}},
\end{aligned}
$$

and consequently,

$$
\left\|E_{\epsilon}(F)\right\|_{\epsilon}=\left\|\phi_{m+1}-\frac{\left(P_{\epsilon}-T_{m}\right)^{\epsilon}}{\epsilon^{m+1}}\right\|_{\epsilon}+o(1), \quad \text { as } \epsilon \rightarrow 0,
$$

since $F \in t^{m+1}$. By Theorem 3.2 .

$$
\inf _{P \in B}\left\|\phi_{m+1}-P\right\|_{0}=\lim _{\epsilon \rightarrow 0}\left\|\phi_{m+1}-\frac{\left(P_{\epsilon}-T_{m}\right)^{\epsilon}}{\epsilon^{m+1}}\right\|_{\epsilon} .
$$

According to 1.3 , there exist constants $\epsilon_{0}, M>0$ such that

$$
\left\|\phi_{m+1}-\frac{\left(P_{\epsilon}-T_{m}\right)^{\epsilon}}{\epsilon^{m+1}}\right\|_{0} \leq M
$$

for all $0<\epsilon \leq \epsilon_{0}$. The equivalence of the norms in $\Pi_{k}^{l}$ implies that the net $\left\{\frac{\left(P_{\epsilon}-T_{m}\right)^{\epsilon}}{\epsilon^{m+1}}\right\}_{0<\epsilon \leq \epsilon_{0}}$ is uniformly bounded on $B$. So, there exists a subsequence of $\left\{\frac{\left(P_{\epsilon}-T_{m}\right)^{\epsilon}}{\epsilon^{m+1}}\right\}_{0<\epsilon \leq \epsilon_{0}}$, which is denoted in the same way, and a polynomial $P_{0}$ such that

$$
\frac{\left(P_{\epsilon}-T_{m}\right)^{\epsilon}}{\epsilon^{m+1}} \text { converges to } P_{0} \text {, uniformly on } B \text {, as } \epsilon \rightarrow 0 .
$$

In consequence, $\mathcal{C} \neq \emptyset$.

On the other hand, if $P_{0} \in \mathcal{C}$, there is a sequence $\epsilon \downarrow 0$ such that $\frac{\left(P_{\epsilon}-T_{m}\right)^{\epsilon}}{\epsilon^{m+1}} \rightarrow P_{0}$. Since $T_{m} \in \mathcal{A}$, we have $P_{\epsilon}-T_{m} \in \mathcal{A}$, and so $P_{0} \in \mathcal{B}$ by Corollary 2.7 Finally, from Property (3) and $(3.8)$ we conclude that

$$
\inf _{P \in B}\left\|\phi_{m+1}-P\right\|_{0}=\lim _{\epsilon \rightarrow 0}\left\|\phi_{m+1}-\frac{\left(P_{\epsilon}-T_{m}\right)^{\epsilon}}{\epsilon^{m+1}}\right\|_{\epsilon}=\left\|\phi_{m+1}-P_{0}\right\|_{0},
$$

i.e., $P_{0}$ is a solution of $(3.7)$.

The following theorem is an extension of [13, Theorem 5.1]. 
Theorem 3.4. Let $\mathcal{A}$ be a non-zero subspace of $\Pi_{k}^{l}$ with $l>m$, and let $\left\{P_{\epsilon}\right\}$ be a net of best approximants of $F$ from $\mathcal{A}$ with respect to $\|\cdot\|_{\epsilon}^{*}$. Assume $m+1=$ $\min \left\{j: 0 \leq j \leq l\right.$ and $\left.A_{j}=\{0\}\right\}, F \in t^{m+1}$ with $T_{m} \in \mathcal{A}$, and set $\phi_{m+1}=T_{m+1}-$ $T_{m}$. If the minimization problem (3.7) has a unique solution $P_{0}$, then $P_{\epsilon} \rightarrow T_{m}+P$, where $P \in \mathcal{A}$ is uniquely determined by the condition $T_{m+1}(P)=P_{0}-T_{m}\left(P_{0}\right)$.

Proof. Since (3.7) has a unique solution $P_{0}$, Theorem 3.3 implies that

$$
\lim _{\epsilon \rightarrow 0} \frac{\left(P_{\epsilon}-T_{m}\right)^{\epsilon}}{\epsilon^{m+1}}=P_{0} .
$$

In consequence, $\partial^{\alpha}\left(P_{\epsilon}-T_{m}\right)(0) \rightarrow 0,|\alpha| \leq m$, and $\partial^{\alpha}\left(P_{\epsilon}-T_{m}\right)(0) \rightarrow \partial^{\alpha} P_{0}(0)$, $|\alpha|=m+1$, as $\epsilon \rightarrow 0$. Therefore

$$
T_{m+1}\left(P_{\epsilon}-T_{m}\right)(x) \rightarrow \sum_{|\alpha|=m+1} \frac{\partial^{\alpha} P_{0}(0)}{\alpha !} x^{\alpha}=: R(x), \quad x \in B, \text { as } \epsilon \rightarrow 0 .
$$

Let $T: \mathcal{A} \rightarrow \Pi_{k}^{m+1}$ be the linear operator defined by $T(P)=T_{m+1}(P)$. As $A_{m+1}=$ $\{0\}$, an analysis similar to that in the proof of Corollary 2.9 shows that $T$ is an injective operator. Since $T(\mathcal{A})$ is a closed subspace and $\left\{T_{m+1}\left(P_{\epsilon}-T_{m}\right)\right\} \subset T(\mathcal{A})$, (3.9) implies that there exists a unique $P \in \mathcal{A}$ such that $T_{m+1}(P)=R$. Hence $T_{m+1}\left(P_{\epsilon}-T_{m}-P\right) \rightarrow 0$ as $\epsilon \rightarrow 0$. As $A_{m+1}=\{0\}$ we see that $\|Q\|:=\left\|T_{m+1}(Q)\right\|_{0}$ is a norm on $\mathcal{A}$, and so $P_{\epsilon} \rightarrow T_{m}+P$ as $\epsilon \rightarrow 0$. Finally, by Theorem 2.6, $\mathcal{B} \subset$ $\Pi_{k}^{m+1}$, and consequently $P_{0}-T_{m}\left(P_{0}\right)=T_{m+1}\left(P_{0}\right)-T_{m}\left(P_{0}\right)=R$. The proof is complete.

Remark 3.5. If $\mathcal{A}$ satisfies the condition (c2), then $\mathcal{A}=\Pi_{k}^{m} \oplus A_{m}$ with $A_{m+1}=$ $\{0\}$. By Corollary 2.9 $\mathcal{B}=\Pi_{k}^{m} \oplus T_{m+1}\left(A_{m}\right)$ and each element $P \in \mathcal{A}$ is uniquely determined by $T_{m+1}(P)$. So, we can rewrite the problem (3.7) in the following (equivalent) form:

$$
\min _{Q+U \in \Pi_{k}^{m} \oplus A_{m}}\left\|\phi_{m+1}-\left(Q+T_{m+1}(U)\right)\right\|_{0}
$$

The following result has been proved in [13, Theorem 5.1] and it is a consequence of Theorem 3.4

Corollary 3.6. Let $\Pi_{k}^{m} \subset \mathcal{A} \subset \Pi_{k}^{l}$ be a non-zero subspace that satisfies the condition (c2) and let $\left\{P_{\epsilon}\right\}$ be a net of best approximants of $F$ from $\mathcal{A}$ with respect to $\|\cdot\|_{\epsilon}^{*}$. Assume $F \in t^{m+1}$. If the minimization problem (3.10) has a unique solution $P_{0}$, then $P_{\epsilon} \rightarrow T_{m}+P$, where $P \in \mathcal{A}$ is uniquely determined by the condition $T_{m+1}(P)=P_{0}-T_{m}\left(P_{0}\right)$.

In the following example we present a function $F \in \bigcap_{m=0}^{\infty} t^{m}$ such that $T_{2}(F) \notin$ $\mathcal{A}$ and the net $\left\{T_{i}\left(P_{\epsilon}\right)\right\}$ does not converge for the same $i>m+1$. 
Example 3.7. Set $B=[-1,1],\|G\|_{\epsilon}=\left(\int_{-1}^{1}|G(x)|^{2} d x\right)^{\frac{1}{2}}, \mathcal{A}=\operatorname{span}\left\{1, x^{2}, x^{3}\right\}$, and $F(x)=x$. So

$$
\|G\|_{\epsilon}^{*}=\left(\frac{1}{\epsilon} \int_{-\epsilon}^{\epsilon}|G(x)|^{2} d x\right)^{\frac{1}{2}}
$$

$A_{0}=A_{1}=\operatorname{span}\left\{x^{2}, x^{3}\right\}, A_{2}=\operatorname{span}\left\{x^{3}\right\}$ and $A_{3}=\{0\}$. Since $T_{1}\left(x^{2}\right)=0$, we observe that the subspace $\mathcal{A}$ does not satisfy the condition (c2). Moreover, an straightforward computation shows that

$$
\frac{\left\|F-T_{0}\right\|_{\epsilon}^{*}}{\epsilon^{0}}=\frac{\sqrt{6}}{3} \epsilon \quad \text { and } \quad \frac{\left\|F-T_{s}\right\|_{\epsilon}^{*}}{\epsilon^{s}}=0, \quad s \in \mathbb{N}
$$

where $T_{0}(x)=0$ and $T_{s}(x)=x$. In consequence, $F \in t^{m}$ for all $m \in \mathbb{N} \cup\{0\}$, and $T_{2}(F) \notin \mathcal{A}$. Since $\int_{-\epsilon}^{\epsilon}\left(x-\frac{7}{5 \epsilon^{2}} x^{3}\right) x^{i} d x=0, i=0,2,3$, then $P_{\epsilon}(x)=\frac{7}{5 \epsilon^{2}} x^{3}$ is the best approximant to $F$ from $\mathcal{A}$ with respect to $\|\cdot\|_{\epsilon}^{*}$. Therefore $T_{i}\left(P_{\epsilon}\right)(x) \rightarrow 0$, for $i=0,1,2$, but $T_{3}\left(P_{\epsilon}\right)(x)$ does not converge, as $\epsilon \rightarrow 0$. So, the best local approximation to $F$ from $\mathcal{A}$ in 0 does not exist, and

$$
\left\|E_{\epsilon}(F)\right\|_{\epsilon}=\frac{\left\|F-P_{\epsilon}\right\|_{\epsilon}^{*}}{\epsilon^{3}}=\frac{2 \sqrt{6}}{15 \epsilon^{2}} \rightarrow \infty, \quad \text { as } \epsilon \rightarrow 0 .
$$

We now give another example which shows that the condition $T_{m} \in \mathcal{A}$ is not necessary for the existence of the best local approximation.

Example 3.8. Set $B,\|\cdot\|_{\epsilon}^{*}$ and $F$ as in Example 3.7 and we consider the subspace $\mathcal{A}=\operatorname{span}\left\{1, x^{2}\right\}$. It is clear that $A_{0}=A_{1}=\operatorname{span}\left\{x^{2}\right\}, A_{2}=\{0\}$ and $\mathcal{B}=\mathcal{A}$. Moreover, we have $F \in t^{2}, T_{1} \notin \mathcal{A}$, and $\mathcal{A}$ does not satisfy the condition (c2) since $T_{1}\left(x^{2}\right)=0$. As $\int_{-\epsilon}^{\epsilon}(x-0) x^{i} d x=0, i=0,2$, then $P_{\epsilon}(x)=0$ is the best approximant to $F$ from $\mathcal{A}$ with respect to $\|\cdot\|_{\epsilon}^{*}$. Therefore, the polynomial 0 is the best local approximation to $F$ from $\mathcal{A}$ in 0 .

\section{REFERENCES}

[1] Billingsley, P., Convergence of Probability Measures, John Wiley \& Sons, New York, 1968. MR 0233396

[2] Chui, C.K., Shisha, O., Smith, P.W., Best local approximation, J. Approx. Theory 15 (1975), no. 4, 371-381. MR 0433101

[3] Chui, C.K., Smith, P.W., Ward, J.D., Best $L_{2}$ approximation, J. Approx. Theory 22 (1978), no. 3, 254-261. MR 0510758

[4] Chui, C.K., Diamond, H., Raphael, L.A., Best local approximation in several variables, J. Approx. Theory 40 (1984), no. 4, 343-350. MR 0740646.

[5] Cuenya, H.H., Ferreyra, D.E., $C^{p}$ condition and the best local approximation, Anal. Theory Appl. 31 (2015), no. 1, 58-67. MR 3338788

[6] Favier, S., Convergence of function averages in Orlicz spaces, Numer. Funct. Anal. Optim. 15 (1994), no. 3-4, 263-278. MR 1272205

[7] Feller, W., An Introduction to Probability and Its Applications, Vol. II, John Wiley \& Sons, New York, 1966. MR 0210154. 
[8] Headley, V.B., Kerman, R.A., Best local approximations in $L^{p}(\mu)$, J. Approx. Theory 62 (1990), no. 3, 277-281. MR 1070281

[9] Macías, R.A., Zó, F., Weighted best local $L^{p}$ approximation, J. Approx. Theory 42 (1984), no. 2, 181-192. MR 0760741.

[10] Maehly, H., Witzgall, Ch., Tschebyscheff-Approximationen in kleinen Intervallen. I. Approximation durch Polynome, Numer. Math. 2 (1960), 142-150. MR 0119002

[11] Walsh, J.L., On approximation to an analytic function by rational functions of best approximation. Math. Z. 38 (1934), no. 1, 163-176. MR 1545445

[12] Wolfe, J.M., Interpolation and best $L_{p}$ local approximation. J. Approx. Theory 32 (1981), no. 2, 96-102. MR 0633695

[13] Zó, F., Cuenya, H.H., Best approximations on small regions. A general approach. In: $A d-$ vanced Courses of Mathematical Analysis II (Granada, 2004), 193-213. World Scientific, Hackensack, NJ, 2007. MR 2343602

F. E. Levis ${ }^{\bowtie}$

Universidad Nacional de Río Cuarto, CONICET, FCEFQyN, Ruta 36 Km 601, X5804BYA

Río Cuarto, Argentina

flevis@exa.unrc.edu.ar

C. V. Ridolfi

Universidad Nacional de San Luis, CONICET, IMASL, Almirante Brown 907, 5700 San Luis, Argentina

ridolfi@unsl.edu.ar

Received: July 4, 2018

Accepted: April 30, 2019 